\title{
Genetic Variability, Correlation, Path Coefficient and Stability Analysis for Yield and its Attributing Traits in Summer Green Gram [Vigna radiata $($ L.) Wilczek] Accessions
}

\author{
V. K. Desai, L. D. Parmar, A. R. Chaudhary* and N. B. Chaudhary \\ Department of Genetics and Plant Breeding, C. P. College of Agriculture, \\ S. D. Agricultural University, Sardarkrushinagar - 385 506, Gujarat, India \\ *Corresponding author
}

Keywords

Green gram, GCV, PCV, Heritability,

Genetic advance,

Correlation, Path

analysis and

stability analysis

Article Info

Accepted:

21 May 2020

Available Online:

10 June 2020

\begin{abstract}
A B S T R A C T
The experiment was conducted to estimate the extent of genetic variability, heritability, genetic advance, correlation coefficient, path coefficient and stability analysis in twenty green gram genotypes for twelve agro-morphological traits using randomized block design (RBD) and 3 replications in summer, 2018. Stability is analysed by creating four environments by varying dates of sowing. Significant differences were found among twenty genotypes for twelve characters in the analysis of variance. Maximum GCV and PCV estimates were found for seed yield per plant, number of pods per plant and number of clusters per plant except in E1. Different values of heritability and genetic advance were observed in different environments among which in E1, heritability was found highest for number of seed per pod, test weight and seed yield per plant, while genetic advance was found highest for number of clusters per plant and seed yield per plant. Seed yield was positively and significantly associated with the yield component at the genotypic and phenotypic level. Two genotypes viz., GM-4 followed by GM-3 was found highest yielder as well as stable genotype among all the genotypes tested.
\end{abstract}

\section{Introduction}

Green gram [Vigna radiata (L.) Wilczek], $2 n=22$ is an important wide-spreading herbaceous, annual, self-pollinated pulse crop in India and occupies third important pulse crop after Chickpea and Pigeon pea. India is the largest producer and consumer of green gram accounting for about 65 per cent of the world's acreage sharing 54 per cent of the world's production (Singh and Singh, 2011).
It is rich in quality proteins, minerals, vitamins and often preferred to other pulses due to its better digestibility and less flatulence problem owing to a lower content of raffinose, stachyose and verbascose (Poehlman, 1991).

It is a cheap and rich source of protein $24.5 \%$ and $59.9 \%$ carbohydrate. It also contains 75 $\mathrm{mg}$ calcium, $8.5 \mathrm{mg}$ iron and $49 \mathrm{mg} \mathrm{R}$ carotene per $100 \mathrm{~g}$ of split dual (Bhowaland 
and Bhowmik, 2014). The protein content of green gram is two to three times more than that of cereals. It is also a rich source of Vitamin B1, Vitamin B2, B-carotene, folic acid, calcium, phosphorous, iron. Ascorbic acid (Vitamin C) is synthesized in sprouted seeds of green gram with increment in niacin, riboflavin and thiamine due to which the majority of salad eaters prefer it as a favourite salad. Among the pulses, green gram occupies an area of about 3.83 million hectares, producing 1.60 million tonnes and productivity of green gram $481 \mathrm{~kg} / \mathrm{ha}$ in India (DE \& S, 2016). In Gujarat, it is cultivated in 1.53 lakh ha with an annual production of 0.86 lakh metric tonnes leading to the average productivity of $561 \mathrm{~kg} / \mathrm{ha}$ (DOA, 2018). Genetic improvement of the crop is largely depending on the magnitude of genetic variability and the extent to which desirable traits are heritable. Genetic variability helps for the choice of the best yield attributes either for selection or for hybridization. It is of utmost importance as it provides the basis for effective selection. Correlation and path analysis facilitates the partitioning of the correlation coefficient into the direct and indirect effect of various character on seed yield or any other attributes and computed as per method given by Wright (1921)-Dewey and $\mathrm{Lu}$ (1959). The evaluation of genotype $\times$ environmental interactions gives an idea of the buffering capacity of the population under study. Thus, Genotype $\times$ Environment interaction certainly plays an important role in the evaluation and execution of breeding programmes as well as predicting the stable genotypes.

\section{Materials and Methods}

The present investigation was carried out at agronomy instructional farm, C. P. College of Agriculture, S. D. Agricultural University, Sardarkrushinagar during the summer season of 2018 in randomized block design (RBD) with three replications and four different environments at research area. The experimental material was comprised of twenty genotypes of green gram collected from the pulse research station, S. D. Agricultural University, Sardarkrushinagar, Gujarat. The data were collected on twelve morphological characters viz., days to flowering, days to maturity, plant height, number of branches per plant, number of clusters per plant, number of pods per cluster, pod length, number of seeds per pod, number of pods per plant, test weight, leaf area and seed yield per plant. Genotypes were sown with the inter-row spacing of $45 \mathrm{~cm}$ and intrarow spacing of $10 \mathrm{~cm}$. All the recommended package of practices was adopted to raise a good crop. Observations on various metric traits were recorded on five randomly selected competitive plants in genotype and replication in mean basis. Analysis of variance for the observations recorded on different characters was carried out as per the standard procedure by Panse and Sukhatme (1985). Genotypic coefficient of variation (GCV) and Phenotypic Coefficient of Variation (PCV) were estimated as per Burton (1952); Heritability $\left(\mathrm{h}^{2}\right)$ and Genetic Advance (GA) were estimated according to Johnson et al., (1955). Phenotypic and genotypic correlation and path coefficients of variation were computed as per the method given by Panse and Sukhatme (1985), Dewey and Lu (1959). Stability analysis was carried out as per procedures of Eberhart and Russel (1966). All the statistical analysis was performed at the department of agricultural statistics using INDOSTAT v8.1. The details of the environments under which the genotypes were grown is given in Table 1 .

\section{Results and Discussion}

All the twelve characters under investigation showed significant variation amid the genotypes signifying their prominence in the 
study of genetic variability (Table 2). The significance of genotype difference indicates the presence of variability for each of the characters among the tested entries (Haile et al., 2015). Availability of variability in the material is of enormous importance for the success of any breeding programme. Mean performance over the different environments are given in Table 3 and pooled analysis of variance mean squares over the environment for different characters are given in Table 4.

\section{Genetic variability}

Estimation for the GCV, PCV, heritability in a broad sense $\left(\mathrm{h}^{2}\right)$ and GA as a per cent of mean are presented in Table 5. High phenotypic coefficient of variation than genotypic coefficients of variation for all the traits in the study might be due to environmental error. The GCV and PCV estimates were found high for seed yield per plant, number of pods per plant and number of clusters per plant except in E1; high PCV for leaf area per plant for all the environments, number of clusters per plant and number of pods per cluster in E1. The PCV and GCV values were low for days to flowering, days to maturity, plant height, test weight for all the environments and number of primary branches per plant in E1, E2 and E3. Low GCV values observed for number of pods per cluster in E2 and leaf area in E3 and number of seeds per pod in E1 and E3; Low GCV and PCV for pod length in E1 and E2.

Similar results were obtained by Pavan et al., (2019), Sreelakhmi et al., (2012), Muralidhara et al., (2015), Varma et al., (2018), Hemavathy et al., (2015) and Anand et al., (2015) for days to flowering; Pavan et al., (2019), Sreelakhmi et al., (2012), Varma et al., (2018) and Hemavathy et al., (2015) for days to maturity; Pavan et al., (2019), Sreelakhmi et al., (2012) and Kumar et al., (2013) for plant height; Pavan et al., (2019) for number of primary branchesper plant and number pods per cluster; Pandiyan ${ }^{\text {a }}$ et al., (2006), Singh et al., (2014), Raje et al., (2000), Hemavathy et al., (2015) and Susmitha and Jayamani (2018) for number of clusters per plant; Ramakrishnan et al., (2018) for number of seeds per pod; Kousar et al., (2007) Raje et al., (2000), Hemevathy et al., (2015), Degefa et al., (2014) and Narasimhula et al., (2013) for number of pods per plant; Pavan et al., (2019) for test weight; Raje et al., (2000), Kousar et al., (2007), Pandiyan ${ }^{\text {b } \text { et }}$ al., (2006), Arshad et al., (2009), Narasimhulla et al., (2013) and Hemavatha et al., (2015) for seed yield per plant.

\section{Heritability and genetic advance}

Most of the traits exhibited moderate to high heritability in almost all the characters in four different environments excluding low heritability for days to flowering, plant height and leaf area in E1 and E3, days to maturity in E2, E3 and E4, number of primary branches per plant and number of pods per cluster in $\mathrm{E} 1, \mathrm{E} 2$, pod length in E1, leaf area in E1, E2 and E3. High heritability for some of the traits like seed yield per plant for all the environments, number of seeds per pod in E1, $\mathrm{E} 2$ and $\mathrm{E} 4$, number of clusters per plant and number of pods per plant in E2, E3 and E4, plant height in E2 and E4, pod length in E3, number of primary branches per plant in E4, test weight in E1 and E4. Seed yield per plant indicates that the improvement for these traits could be made by simple phenotypic selection.

Higher genetic advance as percent of mean was found for number of clusters per plant and seed yield per plant for all the environments. Number of seeds per pod in E2 and E3, number of pods per plant in E2, E3 and E4 and pod length in E1 expressed high genetic advance as percent of mean. Low genetic advance as percent of mean value 
observed for days to flowering, days to maturity for all the environments. Plant height in E1, E3, number of primary branches per plant except for E4, number of pods per cluster in E2, pod length in E1 and E2, test weight only in E4, leaf area only in E3 showed low genetic advance as per cent of mean.

Similar results were found by Sreelakhmi et al., (2012) and Hemavathy et al., (2015) for days to flowering and days to maturity; Pandiyan ${ }^{\mathrm{a}}$ et al., (2006), Pandey et al., (2007) and Kousar et al., (2007) for plant height; Pavan et al., (2019) for number of primary branches per plant; Pandiyan et al., (2006) for number of clusters per plant.

Contradictory results for number of pods per cluster reported by Kousar et al., (2007) and Kumar et al., (2017); Dhole and reddy (2018) for pod length; Pandiyan ${ }^{\mathrm{b}}$ et al., (2006), Yusufzai et al., (2017) and Rahim et al., (2010) for number of pods per plant and number of seeds per pod; Raje et al., (2000), Pandiyan $^{\mathrm{a}}$ et al., (2006), Pandiyan ${ }^{\mathrm{b}}$ et al., (2006), Kumar et al., (2013) and Ahmad et al., (2014) for test weight; Pandiyan ${ }^{\mathrm{a}}$ et al., (2006), Pandiyan ${ }^{\mathrm{b}}$ et al., (2006), Arshad et al., (2009), Rahim et al., (2010) and Degefa et al., (2014) for seed yield per plant.

\section{Variability for pooled over environments}

Variability parameters, heritability and genetic advance for different traits in pooled over environment presented in table 6 revealed that moderate GCV value for number of clusters per plant (13.55\%), number of pods per plant $(11.24 \%)$ and seed yield per plant $(16.02 \%)$. The rest of the traits showed a low value of GCV.

High PCV value for number of clusters per plant $(21.75 \%)$ and leaf area per plant $(22.18 \%)$. The moderate PCV value for number of pods per cluster(18.42\%), number of seeds per pod (11.84\%), number of pods per plant $(15.47 \%)$ and seed yield per plant. The rest of the traits showed a low value of PCV. High heritability for test weight (67.92) and seed yield per plant (70.100). The moderate heritability for days to flowering (30.28), plant height (44.48), number of branches per plant (34.65), number of clusters per plant(38.81), pod length (35.80), number of seeds per pod (51.43) and number of pods per plant (52.78). The rest of the traits showed low heritability. High genetic advance for seed yield per plant (27.64). The moderate heritability value for number of clusters per plant (17.389), number of seeds per pod (12.54) and number of pods per plant (16.82). The rest of the traits showed low genetic advance.

\section{Correlation}

Genotypic and phenotypic correlations presented in Table 7, indicated that seed yield per plant was significant and positively associated with number of primary branches per $\operatorname{plant}\left(\mathrm{r}_{\mathrm{g}}=0.905 * *\right.$ and $\left.\mathrm{r}_{\mathrm{p}}=0.719 * *\right)$, number of clusters per plant $\left(\mathrm{r}_{\mathrm{g}}=0.977 * *\right.$ and $\left.\mathrm{r}_{\mathrm{p}}=0.853^{* *}\right)$, pod length $\left(\mathrm{r}_{\mathrm{g}}=0.890^{* *}\right.$ and $\left.\mathrm{r}_{\mathrm{p}}=0.758 * *\right)$, number of seeds per $\operatorname{pod}\left(\mathrm{r}_{\mathrm{g}}=0.956^{* *}\right.$ and $\left.\mathrm{r}_{\mathrm{p}}=0.823 * *\right)$, number of pods per cluster $\left(r_{\mathrm{g}}=0.644^{* *}\right.$ and $\left.\mathrm{r}_{\mathrm{p}}=0.235^{* *}\right)$, number of pods per plant $\left(\mathrm{r}_{\mathrm{g}}=0.998 * *\right.$ and $\left.\mathrm{r}_{\mathrm{p}}=0.977 * *\right)$, test weight $\left(\mathrm{r}_{\mathrm{g}}=0.649 * *\right.$ and $\left.\mathrm{r}_{\mathrm{p}}=0.486^{* *}\right)$ and leaf area $\left(\mathrm{r}_{\mathrm{g}}=0.515^{* *}\right.$ and $\left.\mathrm{r}_{\mathrm{p}}=0.411^{* *}\right)$ at genotypic and phenotypic level.

Some character showed negative but significant at both the level like days to flowering $\left(\mathrm{r}_{\mathrm{g}}=-0.767 * *\right.$ and $\left.\mathrm{r}_{\mathrm{p}}=-0.505^{* *}\right)$ and plant height $\left(\mathrm{r}_{\mathrm{g}}=-0.863 * *\right.$ and $\left.\mathrm{r}_{\mathrm{p}}=-0.490 * *\right)$. Days to maturity $\left(r_{\mathrm{g}}=-0.410 * *\right.$ and $\left.\mathrm{r}_{\mathrm{p}}=0.030\right)$ showed negative and significant at genotypic level but negative and non-significant at phenotypic level. 
These results are in conformity with Kumar et al., (2017) and Bhutia et al., (2016) for days to flowering, plant height and number of clusters per plant; Patel et al., (2014) and Kumar et al., (2017) for days to maturity and number of primary branches per plant; Ahmad et al., (2012) for number of pods per cluster; Bhutiya et al., (2016), Das et al., (2015) and Kumar et al., (2017), for pod length, number of seeds per pod, number of pods per plant and test weight.

\section{Path co-efficient analysis}

To describe the phenotypic correlation values further path coefficient analysis was done to identify characters having significant direct and indirect effects on grain yield (Table 8). Highest positive direct effect on yield was registered by the number of pods per plant (0.671) followed by number of clusters per plant (0.405). Interestingly, these characters also exhibited a significant positive correlation with yield and therefore, should be considered as important selection criteria for yield improvement of green gram.

While, days to flowering (-0.114), days to maturity (-0.056), number of primary branches per plant (-0.120) and pod length (0.101) had a negative direct effect on seed yield per plant. The residual effect of the path analysis was medium (0.420) suggesting the characters considered in the study were ample and defensible. Similar results were also reported by Kumar et al., (2010) for days to flowering; Bhutiya et al., (2016) for days to maturity, plant height, number of clusters per plant, pod length and number of seeds per pod; Ramakrishnan et al., (2018) and Tabasum et al., (2010) for number of branches per plant, Patel et al., (2014) for number of pods per cluster and number of pods per plant and Das et al., (2015) for the test weight.

\section{Stability analysis}

The pooled analysis of variance (mean square) for stability (Table 9) in respect to the twelve characters of green gram genotypes evaluated across the environments indicated the existence of substantial differences among the four environments in respect to their influence on the expression of twelve characters of 20 green gram genotypes. The mean sum of squares due to genotypes was highly significant for day to flowering $\left(3.38^{* *}\right)$, days to maturity $\left(18.86^{* *}\right)$, plant height $\left(19.73^{* *}\right)$, number of branches per plant $(1.47 * *)$, number of seeds per pod $(1.67 * *)$ and test weight $(0.58 * *)$ which indicating that the genotypes interacted strongly with the environments. Similar results were found by Singh et al., (2014) for number of primary branches per plant, pod length, number of seeds per pod and test weight. For the environment, all the characters except days to maturity and number of pods per cluster indicating that the genotypes interacted strongly with the environments (Fig. 1).

Table.1 Details of the environments under which the genotypes were grown

\begin{tabular}{|l|l|l|}
\hline No. & Date of Sowing & Environments \\
\hline 1. & 12.3 .2018 & Environments $\left(\mathrm{E}_{1}\right)$ \\
\hline 2. & 19.3 .2018 & Environments $\left(\mathrm{E}_{2}\right)$ \\
\hline 3. & 26.3 .2018 & Environments $\left(\mathrm{E}_{3}\right)$ \\
\hline 4. & 2.4 .2018 & Environments $\left(\mathrm{E}_{4}\right)$ \\
\hline
\end{tabular}


Table.2 Analysis of variance mean squares for characters in the individual environment

\begin{tabular}{|c|c|c|c|c|c|c|c|c|c|c|c|c|c|}
\hline Character & Df & $\begin{array}{c}\text { Days to } \\
\text { Flowering }\end{array}$ & $\begin{array}{l}\text { Days to } \\
\text { Maturity }\end{array}$ & $\begin{array}{c}\text { Plant } \\
\text { Height }\end{array}$ & $\begin{array}{c}\text { No. of } \\
\text { Primary } \\
\text { Branches/ } \\
\text { Plant }\end{array}$ & $\begin{array}{l}\text { No. of } \\
\text { Clusters/ } \\
\text { Plant }\end{array}$ & $\begin{array}{l}\text { No. of } \\
\text { Pods/ } \\
\text { Cluster }\end{array}$ & $\begin{array}{c}\text { Pod } \\
\text { Length }\end{array}$ & $\begin{array}{c}\text { No. of } \\
\text { Seeds/ } \\
\text { Pod }\end{array}$ & $\begin{array}{l}\text { No. of } \\
\text { Pods } \\
\text { /plant }\end{array}$ & $\begin{array}{c}\text { Test } \\
\text { weight }\end{array}$ & $\begin{array}{l}\text { Leaf area } \\
\text { per plant }\end{array}$ & $\begin{array}{c}\text { Seed } \\
\text { Yield } \\
\text { /Plant }\end{array}$ \\
\hline \multicolumn{14}{|c|}{ Environment 1} \\
\hline Replication & 2 & 4.55 & 12.82 & 0.52 & $3.49 *$ & 3.44 & $5.60 *$ & 0.22 & 0.26 & 8.79 & 0.0700 & 58202.87 & 6.47 \\
\hline Genotypes & 19 & $3.82 *$ & $36.47 * *$ & $11.58^{*}$ & 1.83 & $11.24 * *$ & $2.55^{*}$ & $0.70 *$ & $2.62 * *$ & $66.67 * *$ & $0.7723 * *$ & $150234.18 *$ & $20.03 * *$ \\
\hline Error & 38 & 1.99 & 12.90 & 5.23 & 1.02 & 2.10 & 1.24 & 0.35 & 0.41 & 14.63 & 0.09 & 68735.79 & 2.37 \\
\hline \multicolumn{14}{|c|}{ Environment 2} \\
\hline Replication & 2 & 4.85 & 1.52 & $21.30 *$ & 1.71 & 2.21 & 0.99 & 0.88 & $1.47 *$ & 6.55 & 0.1272 & 242046.87 & 6.38 \\
\hline Genotypes & 19 & $3.68 *$ & 26.99 & $35.07 * *$ & $1.23 *$ & $11.79 * *$ & 0.94 & $0.73 * *$ & $4.22 * *$ & $163.57 * *$ & $0.64 * *$ & $188832.07^{*}$ & $59.37 * *$ \\
\hline Error & 38 & 1.55 & 17.06 & 5.47 & 0.61 & 1.94 & 0.64 & 0.29 & 0.43 & 11.50 & 0.06 & 99743.74 & 2.18 \\
\hline \multicolumn{14}{|c|}{ Environment 3} \\
\hline Replication & 2 & 1.72 & 10.62 & 19.31 & 0.53 & 1.49 & 0.29 & 0.34 & 0.13 & 3.30 & 0.08 & 4997.42 & 0.17 \\
\hline Genotypes & 19 & $2.75^{*}$ & 32.10 & $12.17 *$ & $1.30 * *$ & $4.22 * *$ & $1.28 * *$ & $1.36 * *$ & $1.23 * *$ & $83.07 * *$ & $0.57 * *$ & 167132.99 & $18.80 * *$ \\
\hline Error & 38 & 1.38 & 22.20 & 6.13 & 0.34 & 0.68 & 0.32 & 0.21 & 0.33 & 3.54 & 0.06 & 151294.48 & 0.57 \\
\hline \multicolumn{14}{|c|}{ Environment 4} \\
\hline Replication & 2 & 3.27 & 15.81 & 15.63 & 0.74 & $1.09 *$ & 0.27 & 0.15 & 0.09 & 0.90 & $0.21 *$ & 56335.33 & 0.23 \\
\hline Genotypes & 19 & $7.67 * *$ & 27.84 & $45.25 * *$ & $2.64 * *$ & $8.17 * *$ & $1.10 * *$ & $2.86 * *$ & $2.55 * *$ & $111.06^{* * *}$ & $0.36 * *$ & $220209.08 * *$ & $25.47 * *$ \\
\hline Error & 38 & 1.62 & 16.11 & 5.49 & 0.44 & 0.26 & 0.29 & 0.23 & 0.30 & 4.10 & 0.05 & 69533.04 & 0.51 \\
\hline
\end{tabular}

**- Significance at 1.0 per cent level of probability, *- Significance at 5.0 per cent level of probability 
Table.3 Mean performance over the different environment

\begin{tabular}{|c|c|c|c|c|c|c|c|c|c|c|c|c|}
\hline Sr. no. & $\begin{array}{c}\text { Days to } \\
\text { Flowering }\end{array}$ & $\begin{array}{l}\text { Days to } \\
\text { Maturity }\end{array}$ & $\begin{array}{l}\text { Plant } \\
\text { Height }\end{array}$ & $\begin{array}{c}\text { No. of } \\
\text { Primary } \\
\text { Branches/ } \\
\text { Plant }\end{array}$ & $\begin{array}{l}\text { No. of } \\
\text { Cluster } \\
\text { s/Plant }\end{array}$ & $\begin{array}{l}\text { No. of } \\
\text { Pods/ } \\
\text { Cluster }\end{array}$ & $\begin{array}{c}\text { Pod } \\
\text { Length }\end{array}$ & $\begin{array}{c}\text { No. of } \\
\text { Seeds/ } \\
\text { Pod }\end{array}$ & $\begin{array}{l}\text { No. of } \\
\text { Pods/ } \\
\text { Plant }\end{array}$ & $\begin{array}{c}\text { Test } \\
\text { Weight }\end{array}$ & $\begin{array}{c}\text { Leaf Area Per } \\
\text { Plant }\end{array}$ & $\begin{array}{l}\text { Seed Yield } \\
\text { /Plant }\end{array}$ \\
\hline Environment 1 & 43.10 & 70.68 & 37.99 & 11.86 & 9.02 & 4.86 & 8.22 & 9.55 & 39.36 & 7.74 & 1542.08 & 17.81 \\
\hline Environment 2 & 43.70 & 70.12 & 42.18 & 10.57 & 7.38 & 4.64 & 7.16 & 7.78 & 30.83 & 7.44 & 1501.26 & 13.45 \\
\hline Environment 3 & 45.17 & 71.42 & 42.34 & 9.48 & 4.98 & 4.58 & 5.24 & 6.44 & 20.98 & 7.05 & 1572.67 & 7.96 \\
\hline Environment 4 & 47.27 & 71.48 & 46.59 & 7.84 & 4.86 & 4.28 & 5.73 & 5.55 & 18.13 & 7.06 & 1298.81 & 6.15 \\
\hline
\end{tabular}

Table.4 A pooled analysis of variance mean squares over the environment for different characters

\begin{tabular}{|c|c|c|c|c|c|c|c|c|c|c|c|c|c|}
\hline $\begin{array}{l}\text { Source of } \\
\text { variation }\end{array}$ & Df & $\begin{array}{c}\text { Days to } \\
\text { Flowering }\end{array}$ & $\begin{array}{l}\text { Days to } \\
\text { Maturity }\end{array}$ & $\begin{array}{c}\text { Plant } \\
\text { Height }\end{array}$ & $\begin{array}{c}\text { No. of } \\
\text { Primary } \\
\text { Branches } \\
\text { /Plant }\end{array}$ & $\begin{array}{c}\text { No. of } \\
\text { Clusters/ } \\
\text { Plant }\end{array}$ & $\begin{array}{l}\text { No. of } \\
\text { Pods/ } \\
\text { Cluster }\end{array}$ & $\begin{array}{c}\text { Pod } \\
\text { Length }\end{array}$ & $\begin{array}{c}\text { No. of } \\
\text { Seeds/ } \\
\text { Pod }\end{array}$ & $\begin{array}{c}\text { No. of } \\
\text { Pods/plant }\end{array}$ & $\begin{array}{c}\text { Test } \\
\text { weight }\end{array}$ & $\begin{array}{l}\text { Leaf area } \\
\text { per plant }\end{array}$ & $\begin{array}{l}\text { Seed Yield } \\
\text { /Plant }\end{array}$ \\
\hline Environment & 3 & $206.37 * *$ & 25.31 & $740.27 * *$ & $173.86^{* *}$ & $242.15^{* *}$ & 3.41 & $110.92 * *$ & $181.60 * *$ & $5639.09 * *$ & $6.65^{* *}$ & $914365.73 * *$ & $1692.27 * *$ \\
\hline Genotype & 19 & $10.16 * *$ & $56.60 * *$ & $59.22 * *$ & $4.42 * *$ & 10.72 & 1.73 & 2.09 & $5.02 * *$ & 121.66 & $1.75^{* *}$ & 220128.66 & 41.05 \\
\hline $\mathbf{G} \times \mathbf{E}$ & 57 & $2.60 *$ & 22.27 & $14.96 * *$ & $0.86^{*}$ & $8.23 * *$ & $1.38 * *$ & $1.19 * *$ & $1.87 * *$ & $100.90 * *$ & $0.19 * *$ & $168759.87 * *$ & $27.53 * *$ \\
\hline Pooled Error & 152 & 1.64 & 17.06 & 5.58 & 0.60 & 1.25 & 0.62 & 0.27 & 0.37 & 8.45 & 0.07 & 97326.76 & 1.40 \\
\hline
\end{tabular}

**- Significance at 1.0 per cent level of probability, *- Significance at 5.0 per cent level of probability 
Table.5 Mean, variability parameters for a different character in an individual environment

\begin{tabular}{|c|c|c|c|c|c|c|c|c|c|c|c|c|}
\hline \multicolumn{7}{|c|}{ Environment-1 } & \multicolumn{4}{|c|}{ Environment-2 } & \multirow[b]{2}{*}{$\mathrm{h}^{2}(\mathrm{~b}) \%$} & \multirow[b]{2}{*}{ GA\% (MEAN) } \\
\hline Parameters & Mean & Range & PCV\% & GCV\% & $\mathrm{h}^{2}(\mathbf{b}) \%$ & GA\% (MEAN) & Mean & Range & PCV\% & GCV\% & & \\
\hline DF & 43.1 & $41.33-45$ & 3.74 & 1.82 & 23.57 & 1.82 & 43.7 & $41.66-45.33$ & 3.44 & 1.93 & 31.38 & 2.22 \\
\hline DM & 70.68 & $65-77.66$ & 6.45 & 3.97 & 37.84 & 5.03 & 70.12 & $64.33-74$ & 6.44 & 2.59 & 16.25 & 2.16 \\
\hline PH & 37.99 & $34.8-44$ & 7.14 & 3.83 & 28.78 & 4.23 & 42.18 & $36.47-47.88$ & 9.28 & 7.45 & 64.35 & 12.3 \\
\hline $\mathbf{B} / \mathbf{P}$ & 11.86 & $10.6-13.53$ & 9.58 & 4.42 & 21.29 & 4.2 & 10.57 & $9.73-11.66$ & 8.55 & 4.32 & 25.52 & 4.49 \\
\hline $\mathrm{C} / \mathrm{P}$ & 9.02 & $6.52-13.65$ & 25.15 & 19.34 & 59.15 & 30.65 & 7.38 & $3.48-10.95$ & 30.96 & 24.55 & 62.9 & 40.12 \\
\hline $\mathrm{P} / \mathrm{C}$ & 4.86 & $3.26-6.04$ & 26.65 & 13.56 & 25.88 & 14.21 & 4.64 & $3.86-5.67$ & 18.53 & 6.83 & 13.57 & 5.18 \\
\hline PL & 8.22 & $7.53-9.21$ & 8.31 & 4.12 & 24.54 & 4.2 & 7.16 & $5.93-8.16$ & 9.23 & 5.38 & 33.97 & 6.46 \\
\hline $\mathbf{S} / \mathbf{P}$ & 9.55 & $6-10.4$ & 11.19 & 8.99 & 64.45 & 14.86 & 7.78 & $4.6-9.46$ & 16.72 & 14.45 & 74.68 & 25.72 \\
\hline $\mathbf{P} / \mathbf{P}$ & 39.36 & $25.21-47.45$ & 14.37 & 10.58 & 54.24 & 16.06 & 30.83 & $19.13-41.90$ & 25.58 & 23.09 & 81.51 & 42.95 \\
\hline TW & 7.74 & $6.68-8.79$ & 7.29 & 6.15 & 71.13 & 10.68 & 7.44 & $6-7.93$ & 6.78 & 5.89 & 75.52 & 10.55 \\
\hline LA & 1542.08 & $1100.23-2005.78$ & 20.08 & 10.69 & 28.33 & 11.72 & 1501.26 & $1150.58-2047.67$ & 23.97 & 11.48 & 22.94 & 11.33 \\
\hline $\mathbf{Y} / \mathbf{P}$ & 17.81 & $10.90-22.11$ & 16.13 & 13.62 & 71.25 & 23.67 & 13.45 & $6.53-19.46$ & 34.28 & 32.47 & 89.73 & 63.36 \\
\hline \multicolumn{7}{|c|}{ Environment-3 } & \multicolumn{4}{|c|}{ Environment-4 } & & \\
\hline Parameters & Mean & Range & PCV\% & GCV\% & $\mathbf{h}^{2}(\mathbf{b}) \%$ & GA\% (MEAN) & Mean & Range & PCV\% & GCV\% & $\mathbf{h}^{2}(\mathbf{b}) \%$ & GA\% (MEAN) \\
\hline DF & 45.17 & $43.66-47$ & 3 & 1.5 & 24.83 & 1.53 & 47.27 & $45-50.33$ & 4.03 & 3.01 & 55.5 & 4.61 \\
\hline DM & 71.42 & $65-76.33$ & 7.07 & 2.54 & 12.95 & 1.89 & 71.48 & $65-79$ & 6.26 & 2.77 & 19.52 & 2.52 \\
\hline PH & 42.34 & $39.16-45.6$ & 6.74 & 3.3 & 24.74 & 3.44 & 46.59 & $43.13-61.06$ & 9.29 & 7.82 & 70.73 & 13.54 \\
\hline $\mathbf{B} / \mathbf{P}$ & 9.48 & $7.46-10.73$ & 8.54 & 5.97 & 48.78 & 8.58 & 7.84 & $6-9.6$ & 13.83 & 10.92 & 62.37 & 17.77 \\
\hline $\mathbf{C} / \mathbf{P}$ & 4.98 & $2.45-6.79$ & 27.39 & 21.8 & 63.38 & 35.76 & 4.86 & $2.19-7.95$ & 35.01 & 33.39 & 90.98 & 65.62 \\
\hline $\mathrm{P} / \mathrm{C}$ & 4.58 & $3.08-5.78$ & 17.45 & 12.32 & 49.85 & 17.92 & 4.28 & $3.24-5.46$ & 17.44 & 12.16 & 48.59 & 17.46 \\
\hline PL & 5.24 & $4.06-6.6$ & 14.71 & 11.79 & 64.25 & 19.47 & 5.73 & $3.43-7.46$ & 18.39 & 16.34 & 78.91 & 29.89 \\
\hline $\mathbf{S} / \mathbf{P}$ & 6.44 & $5.66-7.8$ & 12.3 & 8.48 & 47.56 & 12.05 & 5.55 & $4.4-7.13$ & 18.47 & 15.61 & 71.39 & 27.16 \\
\hline $\mathbf{P} / \mathbf{P}$ & 20.98 & $13.4-30.90$ & 26.13 & 24.54 & 88.23 & 47.49 & 18.13 & $9.54-28.23$ & 34.78 & 32.94 & 89.69 & 64.26 \\
\hline TW & 7.05 & $5.7-7.66$ & 6.8 & 5.8 & 72.66 & 10.18 & 7.06 & $6.23-7.5$ & 5.54 & 4.58 & 68.3 & 7.79 \\
\hline LA & 1572.67 & $1159.06-2014.93$ & 25.16 & 4.62 & 3.37 & 1.75 & 1298.81 & $879.76-1813.13$ & 26.64 & 17.26 & 41.94 & 23.02 \\
\hline $\mathbf{Y} / \mathbf{P}$ & 7.96 & $3.98-12.33$ & 32.37 & 30.96 & 91.48 & 61 & 6.15 & $2.38-10.8$ & 48.29 & 46.87 & 94.19 & 93.7 \\
\hline
\end{tabular}

$\mathrm{DF}=$ Days to flowering $(50 \%), \mathrm{DM}=$ Days to maturity, $\mathrm{PH}=\mathrm{Plant}$ height $(\mathrm{cm}), \mathrm{B} / \mathrm{P}=$ Number of primary branches/plant, $\mathrm{C} / \mathrm{P}=$ number of clusters per plant, $\mathrm{P} / \mathrm{C}=$ number of pods per cluster, $\mathrm{PL}=$ Pod length $(\mathrm{cm}), \mathrm{S} / \mathrm{P}=$ Number of seeds/pod, $\mathrm{P} / \mathrm{P}=$ Number of pods/plant, $\mathrm{TW}=100$-seed weight $(\mathrm{g}), \mathrm{LA}=$ leaf area and $\mathrm{Y} / \mathrm{P}=\mathrm{Seed}$ yield/plant $(\mathrm{g})$ 
Table.6 Mean, range, variability parameters for different traits in pooled over the environment in green gram

\begin{tabular}{|c|c|c|c|c|c|c|c|c|c|c|c|c|}
\hline Parameters & DF & DM & PH & $\mathbf{B} / \mathbf{P}$ & $\mathbf{C} / \mathbf{P}$ & $\mathrm{P} / \mathrm{C}$ & PL & $\mathbf{S} / \mathbf{P}$ & $\mathbf{P} / \mathbf{P}$ & TW & LA & $\mathbf{Y} / \mathbf{P}$ \\
\hline Mean & 44.81 & 70.93 & 42.27 & 9.94 & 6.56 & 4.59 & 6.59 & 7.33 & 27.33 & 7.32 & 1478.70 & 11.34 \\
\hline Range & $\begin{array}{l}43.58- \\
46.91\end{array}$ & $\begin{array}{l}66.33- \\
73.66\end{array}$ & $\begin{array}{l}39.40- \\
47.46\end{array}$ & $\begin{array}{l}8.51- \\
10.91\end{array}$ & $\begin{array}{l}4.87- \\
8.34\end{array}$ & $\begin{array}{l}3.92- \\
5.16\end{array}$ & $\begin{array}{l}5.58- \\
7.2\end{array}$ & $\begin{array}{l}5.45- \\
8.32\end{array}$ & $\begin{array}{l}22.33- \\
32.83\end{array}$ & $\begin{array}{l}6.27- \\
7.92\end{array}$ & $\begin{array}{l}1240.15- \\
1701.75\end{array}$ & $\begin{array}{l}8.83- \\
14.92\end{array}$ \\
\hline PCV\% & 3.42 & 6.36 & 7.50 & 9.65 & 21.75 & 18.42 & 9.88 & 11.84 & 15.47 & 6.21 & 22.18 & 19.14 \\
\hline GCV\% & 1.88 & 2.56 & 5.00 & 5.68 & 13.55 & 6.62 & 5.91 & 8.49 & 11.24 & 5.12 & 6.84 & 16.02 \\
\hline$h^{2}(b) \%$ & 30.28 & 16.18 & 44.48 & 34.65 & 38.81 & 12.92 & 35.80 & 51.43 & 52.78 & 67.92 & 9.51 & 70.10 \\
\hline $\begin{array}{l}\text { GA \% } \\
(\text { MEAN) }\end{array}$ & 2.13 & 2.12 & 6.87 & 6.89 & 17.39 & 4.90 & 7.29 & 12.54 & 16.82 & 8.69 & 4.35 & 27.64 \\
\hline
\end{tabular}

$\mathrm{DF}=$ Days to flowering $(50 \%), \mathrm{DM}=$ Days to maturity, $\mathrm{PH}=\mathrm{Plant}$ height $(\mathrm{cm}), \mathrm{B} / \mathrm{P}=$ Number of primary branches/plant, $\mathrm{C} / \mathrm{P}=$ number of clusters per plant, $\mathrm{P} / \mathrm{C}=$ number of pods per cluster, $\mathrm{PL}=$ Pod length $(\mathrm{cm}), \mathrm{S} / \mathrm{P}=$ Number of seeds/pod, $\mathrm{P} / \mathrm{P}=$ Number of pods/plant, $\mathrm{TW}=100$-seed weight $(\mathrm{g}), \mathrm{LA}=$ leaf area and $\mathrm{Y} / \mathrm{P}=\mathrm{Seed}$ yield/plant $(\mathrm{g})$

Table.7 Genotypic $\left(r_{g}\right)$ (above diagonal) and Phenotypic $\left(r_{\mathrm{P}}\right)$ (below diagonal) correlation coefficients among various characters in green gram

\begin{tabular}{|c|c|c|c|c|c|c|c|c|c|c|c|c|}
\hline Sr. No. & DF & DM & $\mathbf{P H}$ & $\mathbf{B} / \mathbf{P}$ & $\mathbf{C} / \mathbf{P}$ & $\mathrm{P} / \mathrm{C}$ & PL & $\mathbf{S} / \mathbf{P}$ & $\mathbf{P} / \mathbf{P}$ & TW & LA & $\mathbf{Y} / \mathbf{P}$ \\
\hline DF & 1.000 & $0.406 * *$ & $0.765^{* *}$ & $-0.881 * *$ & $-0.684 * *$ & $-0.791 * *$ & $-0.705 * *$ & $-0.752 * *$ & $-0.767 * *$ & $-0.550 * *$ & $-0.500 * *$ & $-0.767 * *$ \\
\hline DM & $0.362 * *$ & 1.000 & $0.401 * *$ & $-0.669 * *$ & $-0.240 * *$ & $-0.321 * *$ & $-0.486 * *$ & $-0.473 * *$ & $-0.481 * *$ & $-0.805 * *$ & $-0.196 * *$ & $-0.410 * *$ \\
\hline PH & $0.353 * *$ & 0.099 & 1.000 & $-0.961 * *$ & $-0.828 * *$ & $-0.770 * *$ & $-0.742 * *$ & $-0.839 * *$ & $-0.883 * *$ & $-0.771 * *$ & $-0.786 * *$ & $-0.863 * *$ \\
\hline $\mathbf{B} / \mathbf{P}$ & $-0.617 * *$ & -0.099 & $-0.466 * *$ & 1.000 & $0.828 * *$ & $0.845^{* *}$ & $0.830 * *$ & $0.943 * *$ & $0.919 * *$ & $0.778 * *$ & $0.606 * *$ & $0.905 * *$ \\
\hline $\mathrm{C} / \mathrm{P}$ & $-0.419 * *$ & 0.003 & $-0.352 * *$ & $0.556 * *$ & 1.000 & $0.518 * *$ & $0.877 * *$ & $0.899 * *$ & $0.972 * *$ & $0.603 * *$ & $0.463 * *$ & $0.977 * *$ \\
\hline $\mathrm{P} / \mathrm{C}$ & -0.102 & -0.068 & $-0.181 * *$ & $0.227 * *$ & $-0.153^{*}$ & 1.000 & $0.623 * *$ & $0.760 * *$ & $0.697 * *$ & $0.790 * *$ & $0.504 * *$ & $0.644 * *$ \\
\hline PL & $-0.514 * *$ & -0.115 & $-0.371 * *$ & $0.672 * *$ & $0.656 * *$ & $0.153^{*}$ & 1.000 & $0.847 * *$ & $0.906^{* *}$ & $0.839 * *$ & $0.162 * *$ & $0.880 * *$ \\
\hline $\mathbf{S} / \mathbf{P}$ & $-0.512 * *$ & -0.076 & $-0.524 * *$ & $0.729 * *$ & $0.622 * *$ & $0.283 * *$ & $0.723 * *$ & 1.000 & $0.966 * *$ & $0.636 * *$ & $0.539 * *$ & $0.956 * *$ \\
\hline $\mathbf{P} / \mathbf{P}$ & $-0.468 * *$ & -0.006 & $-0.480 * *$ & $0.714 * *$ & $0.846 * *$ & $0.249 * *$ & $0.750 * *$ & $0.801 * *$ & 1.000 & $0.708 * *$ & $0.492 * *$ & $0.998 * *$ \\
\hline TW & $-0.344 * *$ & -0.122 & $-0.280 * *$ & $0.523 * *$ & $0.385 * *$ & $0.227 * *$ & $0.547 * *$ & $0.414 * *$ & $0.487 * *$ & 1.000 & $0.263 * *$ & $0.649 * *$ \\
\hline LA & -0.113 & 0.042 & $-0.146^{*}$ & $0.296^{* * *}$ & $0.315^{* *}$ & $0.170 * *$ & $0.168 * *$ & $0.242 * *$ & $0.432 * *$ & $0.174 * *$ & 1.000 & $0.515^{* *}$ \\
\hline $\mathbf{Y} / \mathbf{P}$ & $-0.505 * *$ & -0.030 & $-0.490 * *$ & $0.719 * *$ & $0.853 * *$ & $0.235 * *$ & $0.758 * *$ & $0.823 * *$ & $0.977 * *$ & $0.486 * *$ & $0.411 * *$ & 1.000 \\
\hline
\end{tabular}

* Significant at 5.0 percent, ** Significant at 1.0 percent; $\quad \mathrm{DF}=$ Days to flowering $(50 \%), \mathrm{DM}=\mathrm{Days}$ to maturity, $\mathrm{PH}=\mathrm{Plant}$ height $(\mathrm{cm}), \mathrm{B} / \mathrm{P}=\mathrm{Number}$ of primary branches/plant, $\mathrm{C} / \mathrm{P}=$ number of clusters per plant, $\mathrm{P} / \mathrm{C}=$ number of pods per cluster, $\mathrm{PL}=\mathrm{Pod}$ length $(\mathrm{cm}), \mathrm{S} / \mathrm{P}=\mathrm{Number}$ of seeds $/$ pod, $\mathrm{P} / \mathrm{P}=\mathrm{Number}$ of pods/plant, TW= 100-seed weight $(\mathrm{g}), \mathrm{LA}=$ leaf area and $\mathrm{Y} / \mathrm{P}=$ Seed yield/plant $(\mathrm{g})$. 
Table.8 Genotypic path coefficient analysis of component characters towards seed yield per plant under environment

\begin{tabular}{|l|c|c|c|c|c|c|c|c|c|c|c|}
\hline Character & $\mathbf{D F}$ & $\mathbf{D M}$ & $\mathbf{P H}$ & $\mathbf{B} / \mathbf{P}$ & $\mathbf{C} / \mathbf{P}$ & $\mathbf{P} / \mathbf{C}$ & $\mathbf{P L}$ & $\mathbf{S} / \mathbf{P}$ & $\mathbf{P} / \mathbf{P}$ & TW & $\mathbf{L A}$ \\
\hline $\mathbf{D F}$ & $\mathbf{- 0 . 1 1 4}$ & -0.046 & -0.087 & 0.100 & 0.078 & 0.090 & 0.080 & 0.085 & 0.087 & 0.063 & 0.057 \\
\hline $\mathbf{D M}$ & -0.023 & $\mathbf{- 0 . 0 5 6}$ & -0.022 & 0.037 & 0.013 & 0.018 & 0.027 & 0.026 & 0.027 & 0.045 & 0.011 \\
\hline $\mathbf{P H}$ & 0.085 & 0.044 & $\mathbf{0 . 1 1 1}$ & -0.107 & -0.092 & -0.085 & -0.082 & -0.093 & -0.098 & -0.086 & -0.087 \\
\hline $\mathbf{B} / \mathbf{P}$ & 0.106 & 0.080 & 0.116 & $\mathbf{- 0 . 1 2 0}$ & -0.100 & -0.102 & -0.100 & -0.113 & -0.111 & -0.094 & -0.073 \\
\hline $\mathbf{C} / \mathbf{P}$ & -0.277 & -0.097 & -0.335 & 0.335 & $\mathbf{0 . 4 0 5}$ & 0.210 & 0.355 & 0.364 & 0.394 & 0.244 & 0.188 \\
\hline P/C & -0.008 & -0.003 & -0.007 & 0.008 & 0.005 & $\mathbf{0 . 0 1 0}$ & 0.006 & 0.007 & 0.007 & 0.008 & 0.005 \\
\hline PL & 0.071 & 0.049 & 0.075 & -0.083 & -0.088 & -0.063 & $\mathbf{- 0 . 1 0 1}$ & -0.085 & -0.091 & -0.084 & -0.016 \\
\hline S/P & -0.058 & -0.036 & -0.065 & 0.073 & 0.069 & 0.059 & 0.065 & $\mathbf{0 . 0 7 7}$ & 0.074 & 0.049 & 0.042 \\
\hline P/P & -0.514 & -0.322 & -0.592 & 0.617 & 0.652 & 0.467 & 0.608 & 0.648 & $\mathbf{0 . 6 7 1}$ & 0.475 & 0.330 \\
\hline TW & -0.008 & -0.012 & -0.012 & 0.012 & 0.009 & 0.012 & 0.013 & 0.010 & 0.011 & $\mathbf{0 . 0 1 5}$ & 0.004 \\
\hline LA & -0.028 & -0.011 & -0.044 & 0.034 & 0.026 & 0.028 & 0.009 & 0.030 & 0.028 & 0.015 & $\mathbf{0 . 0 5 6}$ \\
\hline Y/P & -0.767 & -0.410 & -0.863 & 0.905 & 0.977 & 0.644 & 0.880 & 0.956 & 0.998 & 0.649 & 0.515 \\
\hline
\end{tabular}

Residual effect $=0.420 ; \quad \mathrm{DF}=$ Days to flowering $(50 \%), \mathrm{DM}=$ Days to maturity, $\mathrm{PH}=$ Plant height $(\mathrm{cm}), \mathrm{B} / \mathrm{P}=\mathrm{Number}$ of primary branches/plant, $\mathrm{C} / \mathrm{P}=$ number of clusters per plant, $\mathrm{P} / \mathrm{C}=$ number of pods per cluster, $\mathrm{PL}=\mathrm{Pod}$ length $(\mathrm{cm}), \mathrm{S} / \mathrm{P}=$ Number of seeds/pod, $\mathrm{P} / \mathrm{P}=$ Number of pods/plant, $\mathrm{TW}=100$-seed weight $(\mathrm{g})$, $\mathrm{LA}=$ leaf area and $\mathrm{Y} / \mathrm{P}=$ Seed yield/plant $(\mathrm{g})$.

Table.9 A pooled analysis of variance (mean squares) for stability for 12 characters in green gram

\begin{tabular}{|c|c|c|c|c|c|c|c|c|c|c|c|c|c|}
\hline $\begin{array}{l}\text { Source of } \\
\text { variation }\end{array}$ & Df & DF & DM & PH & $\mathbf{B} / \mathbf{P}$ & $\mathbf{C} / \mathrm{P}$ & $\mathbf{P} / \mathrm{C}$ & PL & $\mathbf{S} / \mathbf{P}$ & $\mathbf{P} / \mathbf{P}$ & TW & LA & $\mathbf{Y} / \mathbf{P}$ \\
\hline Genotype & 19 & $3.38 * *$ & $18.86^{* *}$ & $19.73 * *$ & $1.47 * *$ & 3.57 & 0.58 & 0.69 & $1.67 * *$ & 40.57 & $0.58 * *$ & 73376.50 & 13.68 \\
\hline Environment & 3 & $68.79 * *$ & 8.43 & $246.72 * *$ & $57.95 * *$ & $81.28 * *$ & 1.15 & $36.94 * *$ & $60.53 * *$ & $1882.04 * *$ & $2.21 * *$ & $304797.77 * *$ & $564.11 * *$ \\
\hline $\mathbf{G} \times \mathbf{E}$ & 57 & $0.86^{* *}$ & 7.42 & 4.98 & $0.28^{*}$ & 2.74 & 0.45 & 0.39 & 0.62 & 33.66 & 0.06 & 56253.33 & 9.16 \\
\hline $\begin{array}{c}\text { Environment } \\
\text { (Linear) }\end{array}$ & 1 & $206.37 * *$ & $25.30 * *$ & $740.16^{* *}$ & $173.86^{* *}$ & $243.84 * *$ & $3.45^{* *}$ & $110.82 * *$ & $181.59 * *$ & $5646.12 * *$ & $6.65^{* *}$ & $914393.33^{* *}$ & $1692.35^{* *}$ \\
\hline $\mathbf{G} \times \mathbf{E}$ (Linear) & 19 & $1.47 * *$ & 6.96 & $7.47 * *$ & $0.50 * *$ & 2.31 & 0.46 & 0.36 & 0.75 & 18.30 & 0.08 & 49254.58 & 4.54 \\
\hline Pooled deviation & 40 & 0.52 & 7.26 & $3.56^{* *}$ & 0.16 & $2.80 * *$ & $0.43 * *$ & $0.39 * *$ & $0.52 * *$ & $39.28 * *$ & $0.05^{* *}$ & $56765.07 * *$ & $10.90 * *$ \\
\hline Pooled error & 152 & 0.54 & 5.68 & 1.85 & 0.20 & 0.41 & 0.20 & 0.09 & 0.12 & 2.81 & 0.02 & 32442.48 & 0.46 \\
\hline
\end{tabular}

**-Significance at 1.0 per cent level of probability, *- Significance at 5.0 percent level of probability 


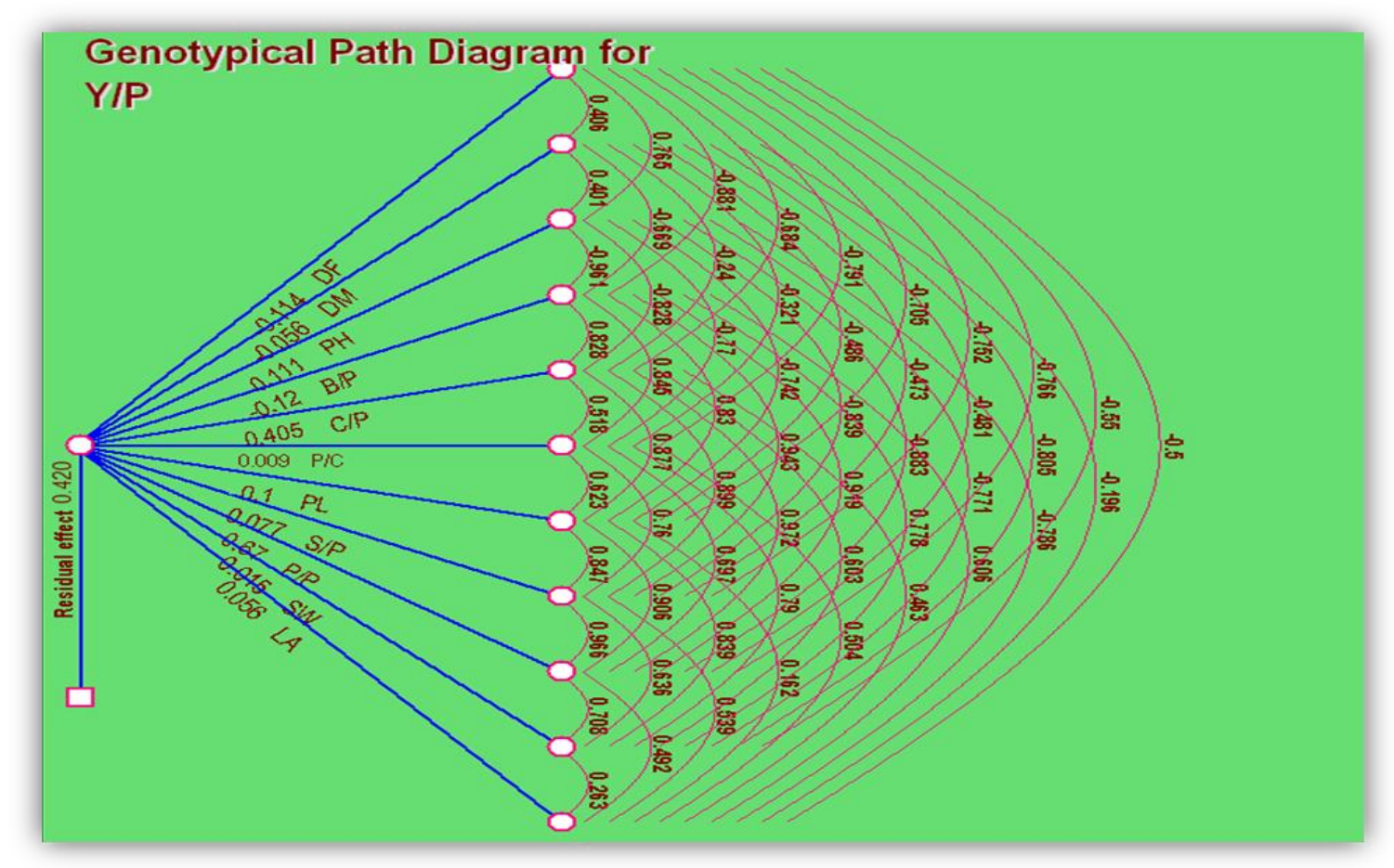

Fig.1 Genotypic path diagram for seed yield per plant

Where, $\mathrm{DF}=$ Days to flowering, $\mathrm{DM}=$ Days to maturity, $\mathrm{PH}=$ Plant height $(\mathrm{cm}), \mathrm{B} \backslash \mathrm{P}=$ Number of primary branches per plant, $\mathrm{C} / \mathrm{P}=$ Number of clusters per plant, $\mathrm{P} / \mathrm{C}=$ Number of pods per cluster, $\mathrm{PL}=$ Pod length $(\mathrm{cm}), \mathrm{S} / \mathrm{P}=$ Number of seeds per pod, P/P $=$ Number of pods per plant , SW $(\mathrm{TW})=100$-seed weight $(\mathrm{g}), \mathrm{LA}=$ Leaf area per plant per plant $\left(\mathrm{cm}^{2}\right)$

Similar results were found by Singh et al., (2014) and Gomashe et al., (2008) for days to maturity, plant height, number of seeds per pod and number of pods per plant was reported. The mean sum of squares due to genotype $\times$ environment was significant for days to flowering $\left(0.86^{* *}\right)$ and number of primary branches per plant $\left(0.28^{*}\right)$ indicating that the genotypes interacted strongly with the environments.

A similar result was also reported by Singh $e t$ al., (2014) for days to flowering and number of primary branches per plant. Environment (linear) was highly significant for all the character indicating that environments differ considerably from one environment to another for these significant characters. Similar results were also reported by Singh et al., (2009) and Singh et al., (2014) for days to maturity, number of seed per pod and number of pods per plant; Patel et al., (2009) and Singh et al., (2014)for plant height, number of primary branches per plant, pod length, test weight and seed yield per plant.

Considering all environments under study, the GCV and PCV estimates were found high for seed yield per plant, number of pods per plant and number of clusters per plant except in E1; high PCV for leaf area per plant for all the environments, number of clusters per plant and number of pods per cluster in E1which indicate ample selection opportunity for these characters. High heritability for the traits like seed yield per plant for all the environments, number of seeds per pod in E1, E2 and E4, number of clusters per plant and number of pods per plant in E2, E3 and E4, plant height in E2 and E4, pod length in E3, number of primary branches per plant in E4, test weight in E1 and E4indicate that the improvement for 
these traits could be made by simple phenotypic selection. Higher genetic advance as percent of mean was found for number of clusters per plant and seed yield per plant for all the environments indicating a predominance of additive gene action and can be improved by simple selection. Path coefficient analysis involving four different environments revealed that number of clusters per plant and number of pods per plant had a high positive direct effect on seed yield per plant which again fortifies the importance of above characters in crop improvement. Two genotypes, GM-4 followed by GM-3 were found highest yielder as well as stable genotype among all the genotypes tested. Since they are having high mean yield, regression near unity and least deviation from regression under four different environmental conditions.

\section{Acknowledgements}

We are thankful to Pulse research station, Sardarkrushinagar for providing resources and Directorate of research, S. D. A. U., along with C. P. College of agriculture for providing research facility and funding to conduct the trial.

\section{References}

Ahmad, H. B., Rauf, S., Rafiq, C. M., Mohsin, A. U., Shahbaz, U. and Sajjad, M. (2014). Genetic variability for yield contributing traits in mung bean (Vigna radiata (L.)Wilczek).Journal of Global Innovations in Agricultural and Social Sciences,2(2): 52-54.

Ahmad, H. M., Ahsan, M., Ali, Q. and Javed, I. (2012). Genetic variability, heritability and correlation studies of various quantitative traits of mung bean (Vigna radiata (L.)Wilczek) at different radiation levels. International Research Journal of Microbiology, 3(11): 352-362.

Anand, G., Anandhi, K. and Paulpandi, V.K. (2015). Genetic variability, correlation and path analysis for yield and yield components in $\mathrm{F}_{6}$ families of Green gram (Vignaradiata (L)Wilczek) under rainfed condition. Electronic Journal of Plant Breeding, 7(2): 434-437.

Arshad, M., Aslam, M. and Irshad, M. (2009). Genetic variability and character association among morphological traits of mung bean (Vigna radiata (L.)Wilczek) genotypes.Journal of Agriculture Research, 47(2): 121-126.

Bhowaland, S. K. and Bhowmik, S. K. 2014. Performance of summer mung bean as affected by variety and date of harvest. Trends in Biosciences, 7(13): 1534-2014.

Bhutia P., Lal, G. M. and Thomas, N. (2016). Studies on genetic variability, correlation and path analysis in green gram (Vigna radiata (L.) Wilczek) germplasm. International Journal of Agriculture Science,6(51): 2267-2272.

Burton, G. W. (1952). Quantitative inheritance in grasses. Proceeding Sixth International Grassland Congress. 1: 277-283.

Das, R. T. and Barua, P. K. (2015). Association studies for yield and its components in green gram. International Journal of Agriculture, Environment and Biotechnology, 8(3): 561-565.

DE and S (2016). Directorate of Economics \&Statistics. Department of Agriculture \&Cooperation, New Delhi.

Degefa, I., Petros, Y. and Andargie, M. (2014). Genetic variability, heritability and genetic advance in mung bean (Vigna radiata (L.)Wilczek) accessions. Plant Science, 1(2): 94-98.

Dewey, D. R. and Lu, K. H. (1959). A correlation and path coefficient analysis of components of crested wheatgrass seed production. Agronomy Journal, 51: 515-518.

Dhole, V. J. and Reddy, K. S. (2018). Genetic analysis and variability studies in mutants induced through electron beam and gammarays in mung bean (Vigna radiata (L.)Wilczek). Electronic Journal of Plant Breeding, 9(1): 304 - 312.

DOA, (2018). Gujarat state area. Production and yield of pulses. Margdarshika. Directorate of Agriculture, Gujarat State, Krishi Bhavan Gandhinagar. 
Eberhart, S. A. and Rusell, W. A. (1966). Stability parameter for comparing varieties. Crop Science, 6: 36-40.

Gomashe, S. S., Patil, J. V., Deshmukh, S. B., Sarode, S. B. and Pise, P. P. (2008). Stability for seed yield and its components in mung bean [Vigna radiata (L.) Wilczek]. Asian Journal of BioScience, 3(1): 111-114.

Haile, J., Legesse, H. and Rao, C. P. (2015). Genetic variability, character association and genetic divergence in barley (Hordeumvulgare L.) genotypes grown at horo District, Western Ethiopia. Science, Technology and Arts Research Journal. 4(2):1-9.

Hemavathy, A. T., Shunmugavalli, N. and Anand, G. (2015). Genetic variability, correlation and path co-efficient studies on yield and its components in mung bean [Vigna radiata (L.)Wilezek].Legume Research,38(4): 442446.

INSEE- The National Institute of Statistics and Economic Studies, France. 2019. Retrieved from

https://www.insee.fr/en/metadonnees/defini tion/c1366

Johnson, H. W., Robinson, H. E. and Comstock, R. E. (1955). Estimation of genetic and environmental variability in soybean. Agronomy Journal. 47(7): 314-318.

Kousar, M., Garard, A., Arifjan. and Singh, A. K. (2007). Genetic variability and correlations studies on yield and its components in mung bean (Vigna radiate (L.)Wilczek). Journal of Agronomy, 6(1): 216-218.

Kumar, G. G., Verma, P. K. and Hari Kesh. (2017). Genetic variability, correlation and path analysis in mung bean [Vigna radiata (L.)Wilczek].International Journal of Current Microbiology and Applied Science, 6(11): 2166-217.

Kumar, K., Yogendra, P., Mishra, B., Pandey, S. and Kumar, R. (2013). Study on genetic variability, correlation and path analysis with grain yield and yield attributing traits in green gram [Vigna radiata (L.)Wilczek]. International Journal of Life Science, 8(4): 1551-1555.

Kumar, N.V., Lavanya, R. G., Singh, S. K. and Pandey, P. (2010). Genetic association and path coefficient analysis in green gram
[Vigna radiata (L.)Wilczek]. AAB Bioflux, 2(3): 251-257.

Muralidhara, Y. S., Lokesh Kumar, B. M., Uday, G. and Shanthala, J. (2015) Studies on genetic variability, correlation and path analysis of seed yield and related traits in green gram [Vigna radiata (L.)Wilczek] International Journal of Agriculture Science and Research, 5(3):125-132

Narasimhulu, R., Naidu N. V., Shanthi Priya, M., Rajarajeswari, V. and Reddy K. H. P. (2013). Genetic variability and association studies for yield attributes in mung bean (Vigna radiata (L.)Wilczek). Indian Journal of Plant Sciences, 2(3): 2319-3824.

Pandey, M. K., Srivastava, N. and Kole, C. R. (2007). Selection strategy for augmentation of seed yield in mung bean (Vigna radiata (L.)Wilczek). Legume Research, 30(4): 243-249.

Pandiyan, M., Subbalakhshmi, B. and Jebaraj, S. $\left(2006^{\mathrm{a}}\right)$. Genetic variability in green gram (Vigna radiata (L) Wilczek). International Journal of Plant Sciences, 1(1): 72-75.

Pandiyan, M., Ganeshram, S., Babu, C., Marimuthu, R. and Bapu, K. $\left(2006^{\mathrm{b}}\right)$. Genetic parameters studies in green gram [Vigna radiata (L.) Wilczek]. Plant Archives, 60(2): 781-784.

Panse, V. G. and Sukhatme, P. V. (1985). Statistical Methods for Agricultural Workers. ICAR, New Delhi. $4^{\text {th }}$ Edition, pp. 97-156.

Patel, S. R., Patel, K. K. and Parmar, H. K. (2014). Genetic variability, correlation and path analysis for seed yield and its components in green gram [Vigna radiata (L.)Wilczek]. International Journal of Life Science, 9(4): 1847-1852.

Pavan, K., Reddy, P. and Mehta, C. M. (2019). Estimation of variability through genetic parameters and identification of superior pure lines for yield attributing traits in green gram [Vigna radiata (L.)Wilczek]. Journal of Pharmacognosy and Phytochemistry, 3: 55-61.

Poehlman, J. M. 1991. The mung bean. Oxford and IBH Publication and company private limited, New Delhi, 375p.

Rahim, M. A., Mia, A. A., Mahmud, F., Zeba, N. and Afrin, K.S. (2010). Genetic variability, 
character association and genetic divergence in mung bean [Vigna radiata (L.)Wilczek]. Plant Omics Journal, 3(1): 16.

Raje, R. S. and. Rao, S. K. (2000). Genetic parameters of variation for yield and its components in mung bean (Vigna radiata (L.) Wilczek) over environments. Legume Research, 23: 211-216.

Ramakrishnan, C. K. D.,Savithramma, D.L. and Vijayabharathi, A. (2018). Studies on genetic variability, correlation and path analysis for yield and yield-related traits in green gram [Vigna radiata (L.) Wilczek]. International Journal Current of Microbiology and Applied Science,7(3): 2753-276.

Singh, C. M., Mishra, S. B., Pandey A. and Madhuri, A. (2014). Morphological characterization and discriminant function analysis in mung bean (Vigna radiata (L.)Wilczek) germplasm. Electronics Journal Plant Breeding, 5(1): 87-96.

Singh, D. P. and Singh, B. B. (2011). Breeding for tolerance to abiotic stresses in mung bean.Journal of Food Legume, 24(2): 8390.

Singh, V., Yadav, R. K., Malik, R. S., Yadav, N. R. and Singh, J. (2013). Stability analysis in mung bean [Vigna radiate (L) Wilczek] nutritional quality and seed yield. Legume Research, 36(1): 56 - 61.

Sreelakshmi, Ch., Reddy Sekhar, M. and Shivani, D. (2012). Studies on genetic variability created through full-sib mating in green gram (Vigna radiata (L.) Wilczek). Electronic Journal of Plant Breeding, 3(4): 973-976.

Susmitha, D. and Jayamani, P. (2018). Genetic variability studies for yield and its contributing traits in green gram [Vigna radiata (L.)Wilczek]. Electronic Journal of Plant Breeding, 9(2): 716-722.

Tabasum, A., Saleem, M. and Aziz, I. (2010). Genetic variability, trait association and path analysis of yield and yield components in mung bean (Vigna radiata (L.)Wilczek). Pakistan Journal of Botany,42(6):39153924.

Varma, P. N., Baisakh, B. and Swain, D. (2018). Study on genetic variability, correlation and path coefficient analysis for yield and component traits in green gram. International Journal of Current Microbiology and Applied sciences, 7(10): 3429-3436.

Wright, S. 1921. Correlation and causation. Journal of Agriculture Research, 20: 557587.

Yusufzai, S. A., Pithia, M. S., RavalLata, J. and VoraZarna, N. (2017). Genetic variability, heritability and genetic advance for seed yield and its components in $\mathrm{F}_{2}$ generations of mung bean (Vigna radiata (L.)Wilczek). International Journal of Pure Applied Bioscience, 5(2): 532-535.

\section{How to cite this article:}

Desai, V. K., L. D. Parmar, A. R. Chaudhary and Chaudhary, N. B. 2020. Genetic Variability, Correlation, Path Coefficient and Stability Analysis for Yield and its Attributing Traits in Summer Green Gram [Vigna radiata (L.) Wilczek] Accessions. Int.J.Curr.Microbiol.App.Sci. 9(06): 2942-2955. doi: https://doi.org/10.20546/ijcmas.2020.906.354 